\title{
UNA OBRA DE ILUMINACIÓN DEL PINTOR JUAN CARLOS RUIZ GIJÓN
}

\author{
AN ILLUMINATED WORK BY THE PAINTER \\ JUAN CARLOS RUIZ GIJÓN
}

\author{
Rosario Marchena Hidalgo \\ Universidad de Sevilla. España \\ romahi@us.es
}

\begin{abstract}
Juan Carlos Ruiz Gijón es un pintor de la segunda mitad del siglo XVII poco conocido pese a su pertenencia a la Academia sevillana de Pintura en la que llegó a detentar el cargo de mayordomo y a su relación, no solo profesional, con directivos de ella como Cornelis Schutt y Matías de Arteaga. La carta de profesión firmada por él de una monja del monasterio de Santa Paula, Agustina de San Pedro, de 1685, añade una obra más a su escaso catálogo.

Palabras clave: Juan Carlos Ruiz Gijón; Academia de Pintura de Sevilla; carta de profesión; monasterio de Santa Paula; iluminación.
\end{abstract}

Juan Carlos Ruiz Gijón is a painter of the second half of the $17^{\text {th }}$ century little known despite his membership in the Sevillian Academy of Painting, in which he came hold the position of butler, and his relationship, not only profesional, with academic directors as Cornelis Schutt and Matías de Arteaga. The vocation letter signed by him of a Monastery of Saint Paula's nun called Agustina de San Pedro in 1685 adds one more work to his acarce catalog.

Keywords: Juan Carlos Ruiz Gijón; Academy of Painting of Seville; vocation letters; Monastery of Saint Paula; illumination.

Juan Carlos Ruiz Gijón es un pintor sevillano del que existen pocos datos documentales y del se conocen pocas pinturas, lo que genera muchas incógnitas sobre su vida y su obra. La primera noticia es la de Ceán Bermúdez diciendo que vivía en Sevilla en 1677 y que él había visto una Inmaculada suya firmada ${ }^{1}$, a la que sigue en el tiempo la de Gestoso que aporta que era mayordomo de la

${ }^{1}$ CEÁN BERMÚDEZ, Juan Agustín: Diccionario histórico de los más ilustres profesores de las bellas artes en España. T. IV. Madrid, 1800, p. 284. 
Academia de Pintura en $1673^{2}$. Buena parte de las noticias que tenemos de él vienen dadas por las investigaciones que se han hecho sobre la figura de su hermano el famoso escultor Francisco Antonio Ruiz Gijón. Nace en Utrera en 1648, es hijo de Lucas Ruiz Gijón y de su mujer María de las Nieves ${ }^{3}$. En 1660 se traslada con toda su familia a Sevilla, a la collación de la Santa Iglesia ${ }^{4}$. En mayo de 1670 Matías de Arteaga le arrienda unas casas en la calle de la Mar, también en la collación de Santa María, ejerciendo de fiador su madre María de las Nieves, ya viuda ${ }^{5}$. En mayo de 1672, viviendo todavía en el Sagrario, se casa con Isabel Hernández y Padilla ${ }^{6}$, y el 26 de ese mismo mes, arrienda una casa en la calle de la Sierpe. El 25 de enero de 1673, viviendo ya en la dicha calle, collación del Salvador, dota a su mujer Isabel Hernández, y el 13 de mayo de 1673 el capitán Miguel de Benavides le arrienda otra casa en la plaza de la Magdalena ${ }^{7}$.

Su paso por la Academia de Pintura solo está avalado documentalmente durante cuatro años. En una fecha indeterminada del curso 1668-1669 recibe un pago de 24 reales, cantidad que se va a repetir en el de 1670-1671 y el día 1 de noviembre de 1671, ya en el siguiente curso, que bajará a doce reales el 5 de junio de 1672 y el 26 de octubre de 1672, siendo ya mayordomo, se le dan 132 reales de vellón de ayuda por los gastos de la academia ${ }^{8}$. Según Gestoso, en 1673 seguía siendo mayordomo ${ }^{9}$, pero cuando, el 5 de noviembre de 1673, 43 profesores del Arte de la Pintura, Escultura y Dorado aprueban unos estatutos generales de la academia, su nombre no aparece entre ellos, pero sí el de su hermano Francisco Antonio $^{10}$. La última noticia conocida hasta este momento, del 17 de abril de 1678, es el testamento de Luisa de Zamora, viuda de Juan Ruiz Gijón, que vive en la collación de San Martín, en la Alameda ${ }^{11}$. Pese a la coincidencia del nombre no se trata de Juan Carlos Ruiz Gijón, que en este momento tendría 30 años y hacía menos de seis que se había casado con Isabel Hernández, porque en 1685

${ }^{2}$ GESTOSO Y PÉREZ, José: Ensayo de un diccionario de los artífices que florecieron en Sevilla desde el siglo XIII al XVIII inclusive. T. I. Sevilla, 1889, p. 232.

${ }^{3}$ BERNALES BALLESTEROS, Jorge: Francisco Antonio Gijón. Sevilla 1982, pp. 20-21.

${ }^{4}$ BAGO Y QUINTANILLA, Miguel; HERNÁNDEZ DÍAZ, José y SANCHO CORBACHO, Antonio: Documentos para la historia del arte en Andalucía. T. II. Sevilla, 1930, p. 216.

${ }_{5}$ KINKEAD, Duncan T.: Pintores y doradores en Sevilla. 1650-1699. Documentos. Bloomington, 2006, p. 485.

${ }^{6}$ BERNALES BALLESTEROS, Jorge: Francisco Antonio Gijón, op. cit., p. 21.

${ }^{7}$ KINKEAD, Duncan T.: Pintores y doradores..., op. cit., pp. 485-486.

8 Ibidem.

${ }^{9}$ GESTOSO Y PÉREZ, José: Ensayo de un diccionario..., op. cit., t. I, p. 232.

${ }^{10}$ HEREZA, Pablo: Corpus Murillo. Biografia y documentos. Sevilla, 2017, p. 102, nota 211.

${ }^{11}$ KINKEAD, Duncan T.: Pintores y doradores..., op. cit., p. 486. 
firma la carta de profesión de Agustina de San Pedro del monasterio de Santa Paula (Figura 1).

Pese a ser un "maestro pintor de imaginería", como se le llama en algunos de los documentos citados, pese a haber estado vinculado a la Academia de Pintura desde 1668 llegando a ocupar un cargo de responsabilidad como es el de mayordomo, su vida y su obra han quedado silenciadas durante mucho tiempo. Tanto es así que hasta hace poco no se conocía más que una sola obra suya: la Inmaculada que cita Ceán Bermúdez ${ }^{12}$, de la colección sevillana de Pedro Desmassieres, firmada en $1671^{13}$, actualmente en la colección de Pedro Romero de Solís de Carmona ${ }^{14}$. En 1972 el desaparecido crítico Torres Martín creyó identificar en una subasta de la sala de arte Durán de Madrid otra obra: la Cabeza de San Pablo degollada que, al parecer, también estaba firmada ${ }^{15}$. Años después, en 1986, ya se conocía también una Cabeza de San Juan Bautista de una colección particular sevillana ${ }^{16}$. A estas obras se sumó en 2002 una Santa María Magdalena de Pazzis de la parroquia de San Pablo de Aznalcázar ${ }^{17}$ y en 2015 dos lienzos de los Santos Juanes del convento de Nuestra Señora del Rosario de El Arahal ${ }^{18}$. En este trabajo se presenta una nueva obra de Juan Carlos Ruiz Gijón: la carta de profesión de Agustina de San Pedro del monasterio de Santa Paula fechada el 3 de junio de $1685^{19}$. Las cartas de profesión son los documentos en los que se recoge la ceremonia solemne por la cual la novicia se compromete para siempre a vivir en el monasterio según sus votos y regla y a morir y ser enterrada en él.

La carta, de papel, encuadernada, de $216 \mathrm{~mm}$ de alto por 148 de ancho, recoge en el texto, realizado con una letra primorosa y combinando la tinta negra con abundantes mayúsculas rojas, el juramento de la profesa:

"Yo Agustina de San Pedro hija de Domingo De Torres y De Doña María días, su mujer hago profesión I prometo Obediencia a Dios i a Santa María y al bien abenturaDo Nuestro Padre S. Gerónimo I A Sr. Dr. D. Joseph de Vaias Provisor I Vicario General Deste arzobispado I $N^{\circ}$ visitador Por el Illmo. I Rmo.

${ }^{12}$ CEÁN BERMÚDEZ, Juan Agustín: Diccionario histórico..., op. cit., t. IV, p. 284.

13 BERNALES BALLESTEROS, Jorge: Francisco Antonio Gijón, op. cit., p. 24.

14 VALDIVIESO GONZÁLEZ, Enrique y SERRERA CONTRERAS, Juan Miguel: La época de Murillo. Antecedentes y consecuentes de su pintura. Catálogo de la Exposición. Sevilla, 1982, p. 150.

${ }^{15}$ BERNALES BALLESTEROS, Jorge: Francisco Antonio Gijón, op. cit., p. 24.

16 VALDIVIESO GONZÁLEZ, Enrique: Historia de la pintura sevillana. Siglos XIII al XX. Sevilla, 1992, p. 237.

${ }^{17}$ FERNÁNDEZ LÓPEZ, José: "Una nueva pintura de Juan Carlos Ruiz Gijón”, Laboratorio de Arte, 15, 2002, pp. 399-402.

18 MARTÍNEZ DEL VALLE, Gonzalo: "Dos nuevas pinturas de Juan Carlos Ruiz Gijón”, Archivo Hispalense, 297-299, 2015, pp. 483-487.

19 AMSPS (Archivo del Monasterio de Santa Paula de Sevilla), Régimen y Gobierno, Profesiones, libro 16, p. 147. 
Sr. D. Jaime de Palafos I Cardona Arsobispo De sevilla Del consejo De S.M.G. i A la Madre Ángela De Santa Isabel Priora deste Monasterio de $N^{\mathrm{a}}$ Madre $\mathrm{S}^{\mathrm{a}}$ Paula...".

Sigue la profesa prometiendo los votos a los que va a estar sujeta y que aún no ha citado -vivir sin propio, en castidad y en perpetua clausura- y guardar la regla de San Agustín. Con toda probabilidad la carta fue escrita también por Juan Carlos Ruiz Gijón, pues su padre era "maestro del arte de enseñar a leer, a escribir y a contar" ${ }^{20}$ y es seguro que debió enseñar a sus hijos el oficio.

La iluminación, que ocupa dos tercios de la carta, es un gran marco que acoge el texto y que más parece un altar que la orla de un documento iluminado. En el centro, arriba, una Virgen en oración con una gran corona enmarcada por dos cortinajes rojos que se recogen con cordones con borlas. A su derecha un busto de San Juan Bautista con manto rojo y cruz astada, a su izquierda el de San Pedro con las llaves. Las tres figuras están inmersas en unas cartelas formadas por hojas trilobuladas que se vuelven sobre sí mismas, de colores rojos, verdes, azules, rosas y lilas muy matizados, una reliquia sin duda de lo que se ha estado haciendo en las miniaturas de distintos libros y documentos en Sevilla y su área de influencia durante la primera mitad del siglo XVII.

A ambos lados del texto, unas columnas salomónicas cubiertas de hojas de parra y racimos de uva reproducen fielmente las del grabado que Matías de Arteaga hizo del antiguo retablo del Cristo de la Expiración de la cofradía del $\mathrm{Mu}$ seo: los fustes cargan sobre altas peanas y sobre los breves capiteles hay cimacios. Los ejemplos de donde pudo extraer el modelo de columna son abundantes. Sin ir más lejos, el cuadro de Los Desposorios de la Virgen del Museo de Bellas Artes de Sevilla, también de Matías de Arteaga, muestra ocho columnas salomónicas recorridas por hojas de parra con los mismos soportes y rematadas por los mismos cimacios que las de la carta de profesión. No es la única vez que Juan Carlos se inspira en Matías de Arteaga, pues su Inmaculada quizás toma el grupo de ángeles que lleva en su base del grabado que a su vez reproduce el cuadro de la Apoteosis de San Francisco de la catedral de Sevilla de Francisco de Herrera el Mozo ${ }^{21}$. La relación de Juan Carlos Ruiz Gijón con Matías de Arteaga es de tipo profesional, al coincidir en la academia donde ambos detentaron cargos, pero también personal según muestra el contrato de arrendamiento citado.

La parte inferior de la orla, de unos $35 \mathrm{~mm}$ de anchura, contiene en el centro una cartela formada por hojas de los mismos elementos, colores y formas que las tres de la parte superior. Dentro de ella la figura de San Agustín. A sus dos lados otras dos cartelas del mismo tipo descrito vacías, reservadas para las firmas del oficiante y de la profesa, que también aparecen en otras cartas como las

20 BERNALES BALLESTEROS, Jorge: Francisco Antonio Gijón, op. cit., p. 25.

${ }^{21}$ CEÁN BERMÚDEZ, Juan Agustín: Diccionario histórico..., op. cit., t. I, p. 77. 
espectaculares de Francisca de San Juan Bautista (25-1-1712) y de Isabel del Buen Suceso $(20-11-1729)^{22}$.

Completa la iluminación de la carta de Agustina de San Pedro la Y(o) que inicia el texto y que se enriquece con una cruz de Malta y unos elementos vegetales.

En la carta abundan los temas significativos, es decir, aquellos que refuerzan el texto, mientras que los decorativos, las cartelas formadas por elementos vegetales, solo son el enmarque de los anteriores. La Virgen y San Juan Bautista probablemente responden a la devoción privada de la profesa; San Pedro es su patrón y representa su nombre lo mismo que San Agustín, aunque en este caso también es la imagen de la regla que rige a las monjas jerónimas; la cruz de Malta alude a la orden y las columnas salomónicas con hojas de parra y racimos de uva a la Eucaristía.

En el borde inferior de la carta una diminuta firma: Gijón (Figura 2). La grafía coincide con las que se conservan de Juan Carlos Ruiz Gijón: en la Inmaculada aparece "Juan Carlos Gijón faciebat"23; en la Santa María Magdalena de Pazzis un papel que sale del cajón de la mesa da el nombre del pintor "Juan Carlos Ruiz Gijón" y la fecha de ejecución, 167024; en los Santos Juanes, el cartel "Juan Carlos Gijón me fecit" lo lleva un loro en el pico en el caso del Bautista y un pájaro blanco y negro en el del Evangelista ${ }^{25}$. Es evidente que al pintor le gustaba firmar sus trabajos y la carta de profesión de Agustina de San Pedro no iba a ser menos.

Cornelis Schutt también iluminó otra carta de profesión para una monja del monasterio de Santa Paula entre octubre de 1682 y junio de 1683. Era la desaparecida de Petronila de San Pedro, hija de Miguel de Usarte, comerciante y tesorero de la hermandad de la Santa Caridad, firmada "Schutt-Pincit-F"26. Ya ha sido puesta de manifiesto la relación existente entre Juan Carlos Ruiz Gijón y Schutt, especialmente a través de la Academia de la que este último era fiscal el 11 de enero de 1660, cónsul en 1663 y $1668^{27}$, y presidente en los cursos 1670-1671 y $1672-73^{28}$. Si es cierto que a partir de 1680 su mala salud le incapacitó para pin$\operatorname{tar}^{29}$, esta no le impidió trabajar en una tarea de menor tamaño como era la iluminación de la carta.

${ }^{22}$ AMSPS, Régimen y Gobierno, Profesiones, libro 16, pp. 161 y 189.

${ }^{23}$ VALDIVIESO GONZÁLEZ, Enrique: Historia de la pintura..., op. cit., p. 150.

${ }^{24}$ FERNÁNDEZ LÓPEZ, José: "Una nueva pintura...", op. cit., p. 400.

${ }^{25}$ MARTÍNEZ DEL VALLE, Gonzalo: "Dos nuevas pinturas...”, op. cit., p. 485

${ }^{26}$ GESTOSO Y PÉREZ, José: Ensayo de un diccionario..., op. cit., t. II, p. 104.

${ }^{27}$ CORZO SÁNCHEZ, Ramón: La Academia del Arte de la Pintura de Sevilla 16601674. Sevilla, 2009, pp. 19, 37 y 56.

${ }^{28}$ HEREZA, Pablo: Corpus Murillo..., op. cit., p. 100.

29 VALDIVIESO GONZÁLEZ, Enrique: La escuela de Murillo. Sevilla, 2018, p. 193. 
Otros muchos pintores no tuvieron inconveniente alguno en iluminar libros y documentos, como Alejo Fernández, Andrés Ramírez ${ }^{30}$ o Diego de Zamora ${ }^{31}$ en el siglo XVI y Francisco Pacheco ${ }^{32}$, Diego Gómez ${ }^{33}$ o Francisco de Herrera el Viejo $^{34}$ en el XVII, porque era un trabajo tan bien pagado, proporcionalmente a su tamaño, como cualquier otro de pintura.

La iluminación de la carta de profesión de Agustina de San Pedro se añade al escaso catálogo de las obras de Juan Carlos Ruiz Gijón y nos muestra otra ocupación para un pintor en la difícil Sevilla de fines del siglo XVII.

Fecha de recepción: 30 de octubre de 2018

Fecha de aceptación: 1 de julio de 2019

${ }^{30}$ MARCHENA HIDALGO, Rosario: Las miniaturas de los libros de coro de la Catedral de Sevilla: el siglo XVI. Sevilla, 1998, pp. 117-121 y 172-220.

${ }^{31}$ GESTOSO Y PÉREZ, José: Ensayo de un diccionario..., op. cit., Apéndices, p. 420.

32 PACHECO, Francisco: Arte de la Pintura. T. II. Madrid, 1956, cap. III, p. 37.

${ }^{33}$ HIDALGO OGAYAR, Juana: "Diego Gómez, pintor-iluminador del siglo XVII", en Velázquez y su tiempo. Madrid, 1991, pp. 339-346.

${ }^{34}$ MARTÍNEZ RIPOLL, Antonio: Francisco de Herrera el Viejo. Sevilla, 1978, pp. 20 y 66. 


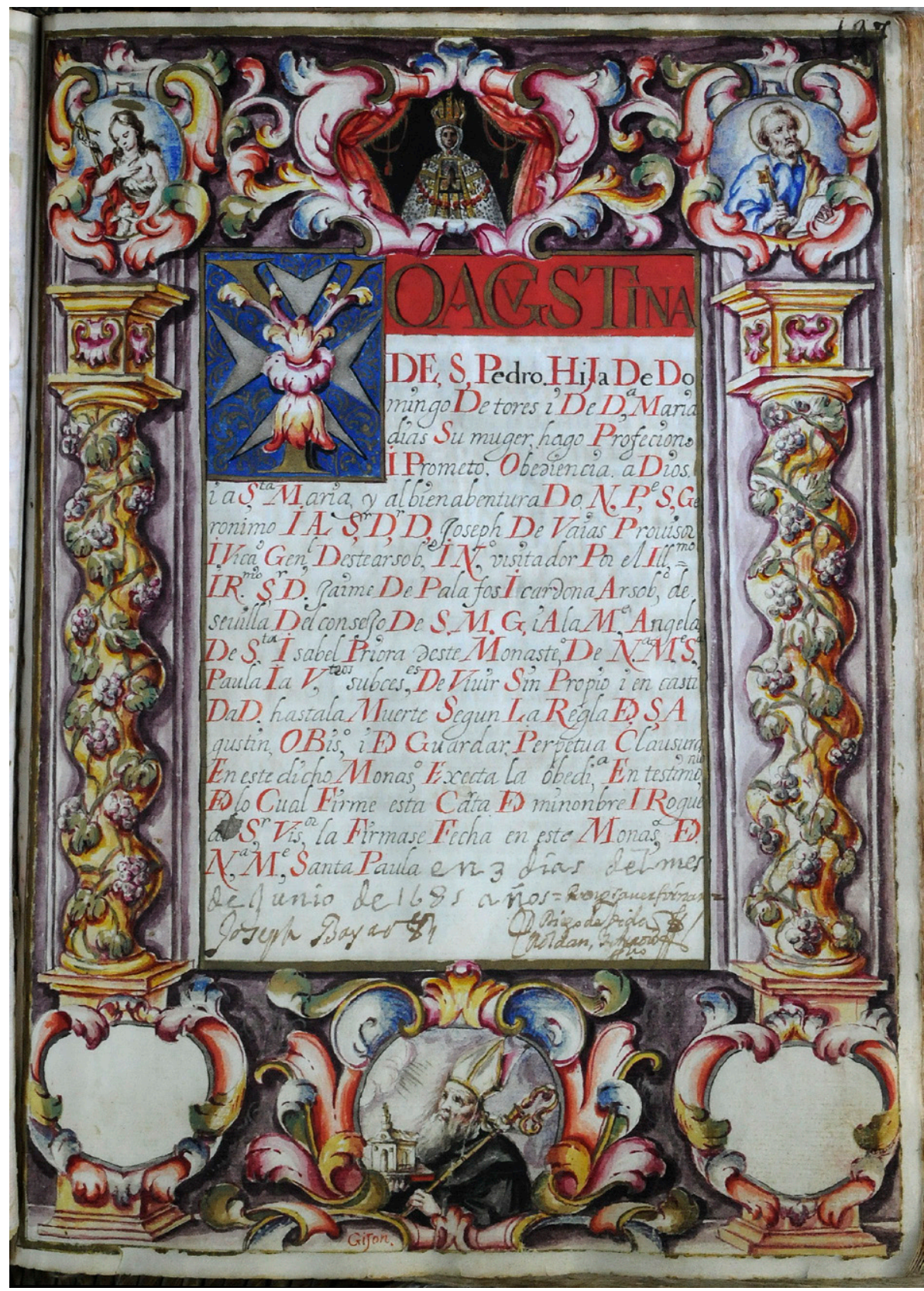

Figura 1. Juan Carlos Ruiz Gijón, Carta de profesión de Agustina de San Pedro, 3-6-1685.

LABORATORIO DE ARTE 31 (2019), pp. 653-660, ISSN 1130-5762 e-ISSN 2253-8305 - DOI http://dx.doi.org/10.12795/LA.2019.i31.39 


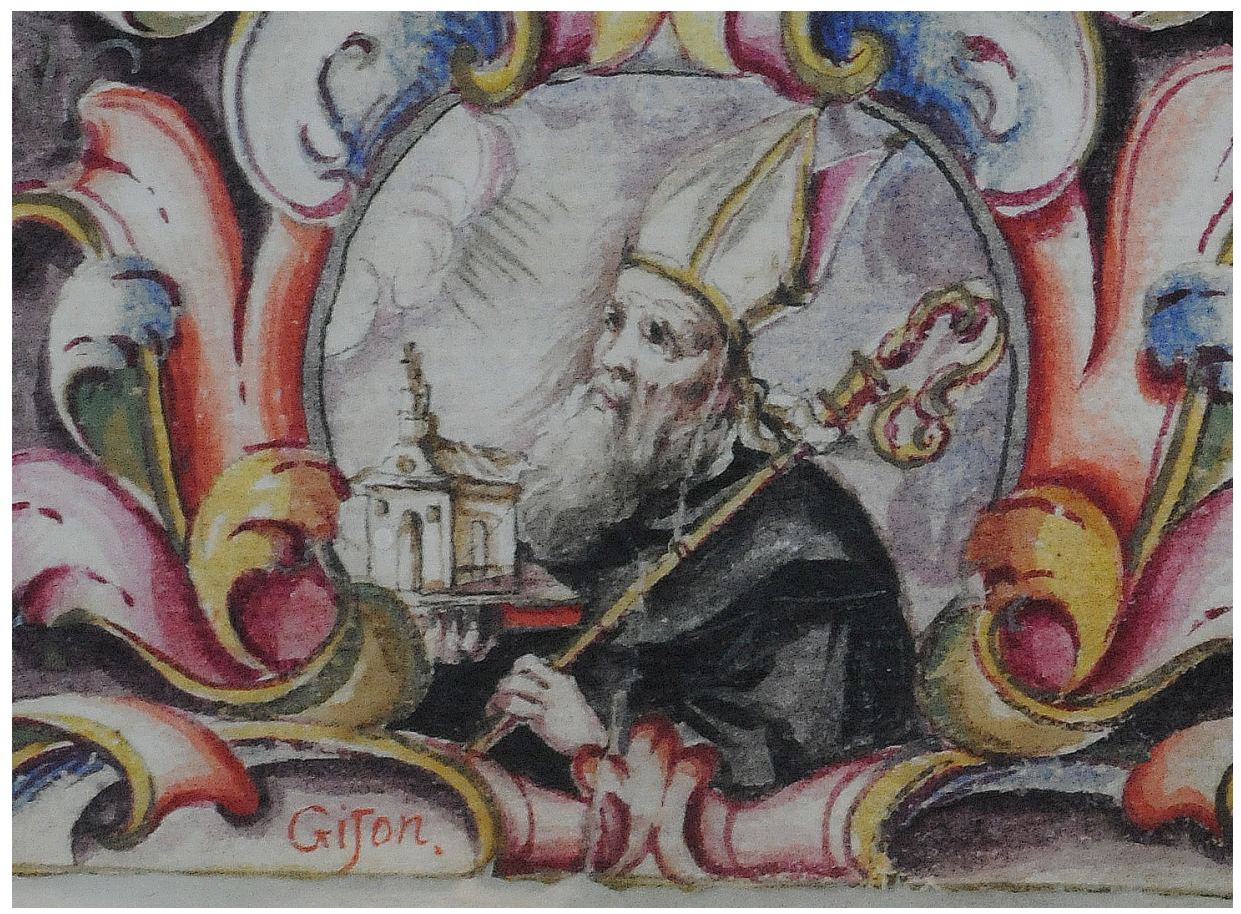

Figura 2. Juan Carlos Ruiz Gijón, Carta de profesión de Agustina de San Pedro, 3-6-1685. Detalle con la firma del autor. 